\title{
Combining SARS-CoV-2 proofreading exonuclease and RNA-dependent RNA polymerase inhibitors as a strategy to combat COVID-19: a high- throughput in silico screen
}

\author{
Dr. Shradha Khater ${ }^{1}$, Dr. Nandini Dasgupta² and Dr. Gautam Das ${ }^{2}$ \\ ${ }^{1}$ Department of Biosciences and Bioengineering, Indian Institute of Technology Bombay, \\ Mumbai, India \\ ${ }^{2}$ miBiome Therapeutics LLP, Mumbai \\ Contact: shradha.khater@iitb.ac.in, gautam@microbiome.in
}

\begin{abstract}
:
SARS-CoV-2 has infected millions of people worldwide. Currently, many clinical trials for drugs against COVID-19 are underway. Viral RNA-dependent RNA polymerase (RdRp) remains the target of choice for prophylactic or curative treatment of COVID-19. Nucleoside analogs are the most promising RdRp inhibitors and have shown effectiveness in vitro as well as in clinical settings. One limitation of such RdRp inhibitors is removal of incorporated nucleoside analogs by SARS-CoV-2 exonuclease (ExoN). Thus, it accomplishes resistance to many of the RdRp inhibitors. We hypothesize that in the absence of highly efficient antivirals to treat COVID-19, combination drugs with RdRp and ExoN inhibitors will be a promising strategy to combat the disease. To repurpose drugs for COVID-19 treatment, 10,397 conformers of 2,240 approved drugs were screened against ExoN domain of nsp14. The molecular docking approach helped us to identify Dexamethasone metasulfobenzoate, Conivaptan, Hesperidin and Glycyrrhizic acid as potential inhibitors of ExoN activity. We recommend further investigation of a combinational therapy using RdRp inhibitors with a repurposed ExoN inhibitor, deciphered through this study, as a potential COVID-19 treatment.
\end{abstract}

\section{INTRODUCTION:}

On December 31, 2019, the World Health Organization (WHO) Office in China was informed that cases of pneumonia of an unknown cause had been detected in Wuhan City, in the Hubei Province of China. The Chinese authorities identified this to be a previously unknown type of coronavirus, Severe acute respiratory syndrome coronavirus 2 (SARS-Cov-2), causing the disease COVID-19. Since the outbreak, the number of confirmed cases of COVID-19 increased rapidly resulting in WHO declaring the disease a pandemic on March 11, 2020. Almost all countries have been affected by this pandemic with millions of confirmed cases and hundreds of thousands of deaths worldwide. Currently there are no approved drugs. Repurposed antivirals are showing good promise in clinical trials. However, there is an urgent need to have a repertoire of repurposed drugs to improve the efficacy and in addition be prepared for drug resistance. Synergistic action of a combination of drugs against SARS-CoV2 can enhance the effectiveness of existing drugs which have shown partial success in clinical 
trials. The class of antivirals which are RNA-dependent RNA polymerase (RdRp) inhibitors like Favirpiravir, Remdesivir, Ribavirin and Galidesivir have been on high priority since the beginning of COVID-19 trials. Trials have been completed/are in progress in many countries. Among these, Remdesivir and Favipiravir has shown promise in different countries. These drugs being nucleoside analogs act either by introducing mutations in the viral RNA or by chain termination during replication. The action of these drugs on viruses which do not have proofreading enzymes have been good [1]. However, SARS-Cov-2 possesses a nonstructural protein nsp14, with amino terminal domain coding for a proofreading exonuclease (ExoN) [2]. ExoN is capable of excising incorporated nucleoside analogs by virtue of its 3'-5' exonuclease proofreading activity. This results in negating the action of these drugs, to varying extents, depending on the type of nucleoside analog chemistry (Ribavirin, 5FU, Remdesivir) (Figure 1) [3, 4]. Hence, in case of repurposed drugs for COVID-19, limitation of efficacy exists. The new generation RdRp inhibitors like Remdesivir have been more effective than Ribavirin and 5FU, in that the excision of these nucleosides by viruses harbouring exoribonuclease has been weaker than Ribavirin and 5FU [5]. The delicate balance between incorporation and excision properties of nucleoside analogs between the RdRp and ExoN decides the fate of the action of RdRp-based antivirals.

ExoN inactivation was found to confer a "mutator phenotype," as was evident from a 15- to 21 -fold increase in mutation frequency - relative to the wild-type (wt) control - during replication and passaging in cell culture $[6,7]$. There is evidence that in ExoN mutant background, Remdesivir has 4.5-fold more efficacy [8] and Ribavirin has 200-fold higher efficacy [3] when compared to a wild type ExoN viral genome. The mutagenesis results are supported by sequencing analyses too [6]. This provides a clear rationale to use a combination of antivirals Favirpiravir/Remdesivir/Ribavirin/Galidesivir and SARS-CoV-2 ExoN inhibitors. Currently there is no drug available to inhibit nsp14. Detailed molecular docking studies to find small molecules/peptides/natural molecules that have the potential to inhibit nsp14 ExoN, are required urgently [9]. This would potentially lead to improve the current efficacy of the RdRp inhibitors in COVID-19. It is interesting to note that cornonaviruses lacking ExoN are susceptible to lethal mutagenesis [4]. Crystal structure of SARS-CoV nsp14-nsp10 [10] provides opportunities for molecular docking of the ExoN domain of nsp14 to different available drugs.

In this study, we propose that a combinatorial therapy with one drug from Favirpiravir/Remdesivir/Ribavirin/Galidesivir and an inhibitor of ExoN would be effective in increasing the efficacy of the above RdRp inhibitors (Figure 1). In an effort to repurpose drugs for COVID-19 treatments, we have performed molecular docking of 10,397 approved drug 
conformers on ExoN domain of SARS-CoV-2 nsp14. Three known antivirals Conivaptan, Hesperidin and Glycyrrhizic acid show promise based on the docking results and their known inhibitory effects on beta coronaviruses in vitro [11] and in patients [12]. Molecular docking result indicated Dexamethasone metasulfobezoate as lowest energy binding partner of ExoN domain. Interestingly, dexamethasone was recently shown to reduce fatality in COVID-19 patients [13]. Therefore, repurposing Dexamethasone metasulfobenzoate, Conivaptan, Hesperidin and Glycyrrhizic acid as potential inhibitors of proof-reading ExoN (nsp14) and using them in conjunction with RdRp inhibitors could lead to potentially high level of antiviral activity and a promising therapy for COVID-19.

\section{RESULTS:}

\section{SARS-CoV-2 ExoN domain}

SARS-CoV-2 nsp14 is a multidomain protein. The $\mathrm{N}$-terminal domain functions as proofreading exoribonuclease and the C-terminal is a methyl transferase. SARS-CoV-2 nsp14 shares $95.07 \%$ amino acid sequence identity (over complete protein length) with SARS-CoV nsp14. ExoN domain of SARS-CoV nsp14 resembles DEDD-type exonucleases [10]. The DEDD superfamily members are defined by presence of three canonical motifs - DXE (Motif I), W(X)4EL (Motif II), and DAIMTR (Motif III) [8]. Presence of DEED instead of DEDD and an additional $\mathrm{H}$ makes the SARS-CoV ExoN a DEEDh type exonuclease [5]. In SARS-CoV-2, the catalytic residues - Asp90, Glu92, Glu191, His268, and Asp273, and the canonical motifs are conserved (Supplementary Figure S1). 3D model of SARS-CoV-2 nsp14 was built using SARS-CoV nsp14 (PDB ID: 5C8S) as template. A grid comprising of the three conserved motifs was used for docking.

\section{Molecular Docking}

10,397 conformers generated from 2,240 approved small molecule drugs were screened using AutoDOCK VINA. Based on binding affinity energy, top 20 binding poses were selected for further analysis (Table 1, Figure 2). All 20 poses interact with catalytic residues. Dexamethasone metasulfobenzoate binds to the catalytic site of ExoN with binding energy of -8.7 kCal/mol. Conivaptan, Dutasteride, Hesperidin, Lumacaftor and Glycyrrhizic acid bind ExoN active site with slightly higher energy of $-8.6 \mathrm{kCal} / \mathrm{mol}$. Interaction of ExoN domain with 12 unique drug molecules, corresponding to top 20 poses, was studied and are depicted in Table 2. Most of the analysed poses interact with at least 3 out of 5 catalytic residues (Figures

3 \& 4). Hesperidin is the only drug that interacts with all five catalytic residues. Hesperdin and Glycyrrhizic acid has 4 and 3 ExoN catalytic residues respectively, within hydrogen bonding distance (Figure 4). Binding of these drugs to ExoN catalytic residues might potentially cause obstruction of substrate binding and catalysis. 
Remdesivir, an investigational drug for treatment of Ebola, was shown to inhibit SARS-CoV-2 RNA-dependent RNA polymerase, inhibiting RNA synthesis[14]. As Remdesivir is a drug without anti-ExoN activity, it was used as a negative control. The binding energy of Remdesivir with ExoN is $-6.0 \mathrm{kCal} / \mathrm{mol}$. Hence, the binding energy of Remdesivir is much higher than the top 20 poses obtained using approved drugs (Table 1).

\section{DISCUSSION:}

COVID-19, an infectious respiratory illness, is caused by a novel strain of coronavirus SARSCoV-2. Currently, there are no approved drugs or vaccines. Drugs targeting RdRp like Favipiravir, Remdesivir, Ribavirin and Galidesivir have shown promise in SARS-CoV-2 and few other strains of coronavirus. Nucleoside analogs - Remdesivir and Favipiravir have been authorized for emergency use in the treatment of COVID-19 in different countries. These RdRp inhibitors act by competing with host nucleoside substrates for incorporation in nascent RNA being synthesized [15]. The mis-incorporated analogs either cause a premature chain termination or mutation in RNA (Figure 1). ExoN domain of nsp14 in CoVs is known to excise mis-incorporated analogs [3]. The excision action of ExoN decreases the efficacy of nucleoside analog like Ribavirin in vivo [3]. Decrease in efficacy of Remdesivir has been speculated due to ExoN activity [14]. A 100-fold increase in Remdesivir efficacy was seen in ExoN mutant of murine hepatitis virus (MHV), a betacoronavirus [16]. It's been hypothesized that simultaneous inhibition of RdRp and ExoN in CoVs could be an effective therapeutic strategy [17].

Hence, in silico drug screening method was used to search for potential inhibitor of ExoN. 10,397 conformers from 2,240 approved drugs were screened against SARS-CoV-2 nsp14 containing the ExoN domain. AutoDOCK VINA screening results indicated Dexamethasone metasulfobenzoate to be the top binder, and Conivaptan, Dutasteride, Hesperidin, Lumacaftor and Glycyrrhizic acid to be close second. All six compounds showed extensive interaction with nsp14 residues, especially the catalytic residues of ExoN domain (Table 2). Hence, we hypothesize that these drugs might occlude ExoN catalytic site, thereby inhibiting the proofreading activity (Figure 1).

Interestingly, few of the approved drugs that bind with ExoN catalytic site in silico- Conivaptan, Hesperidin and Glycyrrhizic acid - have shown anti-viral activity in earlier studies. Conivaptan, a non-peptide inhibitor of vasopressin, has shown in vitro efficacy against feline infectious peritonitis coronavirus (FIPV) and human coronavirus OC43 (HCoV-OC43) [18]. Conivaptan showed inhibitory effect against Dengue and Zika virus at high concentrations. Inhibitory activity of Hesperidin against Influenza A virus was reported by Dong et al [19]. Its antivirus response was linked to increase in cell-autonomous immune responses (enhanced 
expression of primary and secondary genes). In addition, Hesperidin inhibited export of viral ribonucleoproteins. Hesperitin, aglycone form of hesperidin, was shown to inhibit cleavage activity of SARS-CoV 3C-like protease.

Glycyrrhizic acid, an anti-tumoral, anti-inflammatory drug, has in vitro inhibitory effects on a broad range of viruses like flaviviruses [20], herpes viruses and human immunodeficiency virus [21]. Glycyrrhizic acid has been used for treatment of SARS-CoV [12] and chronic hepatitis virus in patients [22]. Hence, there are varied degree of evidence in support of antiviral use of Conivaptan, Hesperidin and Glycyrrhizic acid as antiviral. Our docking result combined with evidence in support of antiviral use of Conivaptan, Hesperidin and Glycyrrhizic acid, indicate their potential as SARS-CoV-2 ExoN inhibitor. When used in a combination with $\mathrm{RdRp}$ inhibitors, the higher concentrations reported for the repurposed ExoN inhibitors to exert antiviral activity could be minimised. In addition, the higher concentrations required for RdRp inhibitors to exert their action in the presence of exonuclease activity like in SARS-Cov-2 can also be reduced as dual action of $\mathrm{RdRp}$ and ExoN inhibition in parallel should ideally require lower concentrations of the respective drugs.

In a randomized controlled trial at Oxford University (Recovery Trial), Dexamethasone, a glucocorticoid, has been recently shown to reduce fatality by third in critically ill COVID-19 patients requiring ventilator support [13]. Dexamethasone acts by reducing inflammation and countering body's overreaction called cytokine storm. Here we propose use of Dexamethasone metasulfobenzoate as an inhibitor of ExoN. Dexamethasone's antiviral activity is predicted to be pronounced in combination with RdRp inhibitors, according to our model (Figure 1). Use of these potential ExoN inhibitors in combination with nucleoside analog targeting RdRp, warrants further investigation in clinical settings.

A combination of RdRp inhibitors and ExoN inhibitors, in addition to the increased efficacy, would also possibly avert drug resistance generated from mutations in ExoN to enhance proofreading of nucleoside analogs in RdRp inhibition.

Strategy to use combination of RdRp inhibitor and antisense/RNAi technology: In addition to drug candidates for nsp14, it is conceivable that a combination of RdRp inhibitor and an antisense/RNAi strategy to inhibit nsp14 can lead to enhanced success in clinical trials. Combined with nasal delivery systems, this can hold promise in developing new combination antiviral therapies for COVID-19. An FDA-approved technology for antisense antiviral technology exists: Fomivirsen (Vitravene®, ISIS-2922), ASO antiviral drug with phosphodiester linkages that are modified by replacing a non-linking oxygen with a sulfur atom (phosphorothioate modification). An FDA-approved technology for RNAi also exists: Onpattro, an RNAi therapeutic for people with a rare and deadly genetic disorder called hereditary transthyretin-mediated amyloidosis. Morpholino oligos for coronavirus have been used experimentally [23]. 
We conclude that even if the repurposed ExoN inhibitors identified in this study are incomplete in isolation, the synergistic effect with RdRp inhibitors will make it a worthwhile case for clinical trials and treatments for COVID-19. These drugs have been extensively used on patients and their safety profiles are well known. Any incremental value in the efficacy of antivirals like Remdesivir, Favipiravir and Ribavirin would lead to huge implications for COVID-19-related healthcare burden globally.

\section{METHODS:}

\section{Homology modelling of ExoN}

Amino acid sequence of SARS-CoV-2 ExoN/nsp14 (P0DTD1) was used as a target sequence to build 3D-model using SWISS-MODEL webserver [24]. Nsp14 from SARS-CoV (PDB ID: $5 \mathrm{C} 8 \mathrm{~S}$, chain $\mathrm{B}$ ) had high sequence identity to target sequence $-95.07 \%$. This $3 \mathrm{D}$ structure was used as a template for building SARS-CoV-2 nsp14 model. The model quality was assessed using QMEAN score. The QMEAN score for generated model was -3.14. Scores above -4 indicates a good model.

\section{Virtual screening using AutoDock VINA}

2D structures of approved small molecule drugs were downloaded from DrugBank in sdf format (2454 structures) [25]. The structures were converted to 3D format using OpenBabel -gen3d option [26, 27]. Few of the drug molecule structures showed error at this stage. 3D structures of these molecules were downloaded from other sources. Multiple conformers for each 3D structure were generated using OpenBabel.

Docking grid was defined encompassing the conserved motives of ExoN domain (see result). A total of 10,397 conformers from 2,240 compounds were subjected to virtual screening using the AutoDOCK VINA tool on SARS-CoV-2 nsp14 [28]. 20 poses were generated for each round. Compound-nsp14 interactions were visualized using LigPlot ${ }^{+}$v2.1 [29].

\section{Acknowledgement:}

Authors would like to acknowledge Sandeep Chakraborty and Dr. Petety V. Balaji for reviewing the manuscript. SK would like to acknowledge DST for DST-WOSA funding (SR/WOS-A/LS141/2018 (G)). We acknowledge SpaceTime-2 supercomputing facility at IIT Bombay for the computing time.

\section{Conflict of Interest:}

Authors declare no conflict of interest 


\section{References:}

1. Warren, T.K., et al., Therapeutic efficacy of the small molecule GS-5734 against Ebola virus in rhesus monkeys. Nature, 2016. 531(7594): p. 381-5.

2. Bouvet, M., et al., RNA 3'-end mismatch excision by the severe acute respiratory syndrome coronavirus nonstructural protein nsp10/nsp14 exoribonuclease complex. Proc Natl Acad Sci U S A, 2012. 109(24): p. 9372-7.

3. Ferron, F., et al., Structural and molecular basis of mismatch correction and ribavirin excision from coronavirus RNA. Proc Natl Acad Sci U S A, 2018. 115(2): p. E162-E171.

4. Smith, E.C., et al., Coronaviruses lacking exoribonuclease activity are susceptible to lethal mutagenesis: evidence for proofreading and potential therapeutics. PLoS Pathog, 2013. 9(8): p. e1003565.

5. Ogando, N.S., et al., The Curious Case of the Nidovirus Exoribonuclease: Its Role in RNA Synthesis and Replication Fidelity. Front Microbiol, 2019. 10: p. 1813.

6. $\quad$ Eckerle, L.D., et al., Infidelity of SARS-CoV Nsp14-exonuclease mutant virus replication is revealed by complete genome sequencing. PLoS Pathog, 2010. 6(5): p. e1000896.

7. $\quad$ Eckerle, L.D., et al., High fidelity of murine hepatitis virus replication is decreased in nsp14 exoribonuclease mutants. J Virol, 2007. 81(22): p. 12135-44.

8. Shannon, A., et al., Remdesivir and SARS-CoV-2: Structural requirements at both nsp12 RdRp and nsp14 Exonuclease active-sites. Antiviral Res, 2020. 178: p. 104793.

9. Senanayake, S.L., Drug repurposing strategies for COVID-19. Future Drug Discovery, 2020. 2(2).

10. Ma, Y., et al., Structural basis and functional analysis of the SARS coronavirus nsp14-nsp10 complex. Proc Natl Acad Sci U S A, 2015. 112(30): p. 9436-41.

11. De Clercq, E., Potential antivirals and antiviral strategies against SARS coronavirus infections. Expert Rev Anti Infect Ther, 2006. 4(2): p. 291-302.

12. Hoever, G., et al., Antiviral activity of glycyrrhizic acid derivatives against SARS-coronavirus. J Med Chem, 2005. 48(4): p. 1256-9.

13. Ledford, H., Coronavirus breakthrough: dexamethasone is first drug shown to save lives, in Nature Briefing2020, natureresearch.

14. Gordon, C.J., et al., The antiviral compound remdesivir potently inhibits RNA-dependent RNA polymerase from Middle East respiratory syndrome coronavirus. J Biol Chem, 2020. 295(15): p. 4773-4779.

15. $\mathrm{Xu}, \mathrm{X}$., et al., Molecular model of SARS coronavirus polymerase: implications for biochemical functions and drug design. Nucleic Acids Res, 2003. 31(24): p. 7117-30.

16. Agostini, M.L., et al., Coronavirus Susceptibility to the Antiviral Remdesivir (GS-5734) Is Mediated by the Viral Polymerase and the Proofreading Exoribonuclease. mBio, 2018. 9(2).

17. Pruijssers, A.J. and M.R. Denison, Nucleoside analogues for the treatment of coronavirus infections. Curr Opin Virol, 2019. 35: p. 57-62.

18. Yang, C.W., et al., Repurposing old drugs as antiviral agents for coronaviruses. Biomedical Journal, 2020. Advance online publication.

19. Dong, W., et al., A dual character of flavonoids in influenza $A$ virus replication and spread through modulating cell-autonomous immunity by MAPK signaling pathways. Sci Rep, 2014. 4: p. 7237.

20. Crance, J.M., et al., Interferon, ribavirin, 6-azauridine and glycyrrhizin: antiviral compounds active against pathogenic flaviviruses. Antiviral Res, 2003. 58(1): p. 73-9.

21. Lin, J.C., Mechanism of action of glycyrrhizic acid in inhibition of Epstein-Barr virus replication in vitro. Antiviral Res, 2003. 59(1): p. 41-7.

22. Miyake, K., et al., Efficacy of Stronger Neo-Minophagen C compared between two doses administered three times a week on patients with chronic viral hepatitis. J Gastroenterol Hepatol, 2002. 17(11): p. 1198-204. 
23. Burrer, R., et al., Antiviral effects of antisense morpholino oligomers in murine coronavirus infection models. J Virol, 2007. 81(11): p. 5637-48.

24. Schwede, T., et al., SWISS-MODEL: An automated protein homology-modeling server. Nucleic Acids Res, 2003. 31(13): p. 3381-5.

25. Wishart, D.S., et al., DrugBank: a comprehensive resource for in silico drug discovery and exploration. Nucleic Acids Res, 2006. 34(Database issue): p. D668-72.

26. O'Boyle, N.M., et al., Open Babel: An open chemical toolbox. J Cheminform, 2011. 3: p. 33.

27. Yoshikawa, N. and G.R. Hutchison, Fast, efficient fragment-based coordinate generation for Open Babel. J Cheminform, 2019. 11(1): p. 49.

28. Trott, $O$. and A.J. Olson, AutoDock Vina: improving the speed and accuracy of docking with a new scoring function, efficient optimization, and multithreading. J Comput Chem, 2010. 31(2): p. 455-61.

29. Laskowski, R.A. and M.B. Swindells, LigPlot+: multiple ligand-protein interaction diagrams for drug discovery. J Chem Inf Model, 2011. 51(10): p. 2778-86. 
Figures and Tables:

Table 1: Screening results of twenty conformers with lowest binding energies. Binding energy is expressed as $\mathrm{kcal} / \mathrm{mol}$

\begin{tabular}{|c|c|c|c|}
\hline Drug Bank ID & Conf ID & Name & Affinity (kCal/mol) \\
\hline DB14703 & 1 & Dexamethasone metasulfobenzoate & -8.7 \\
\hline DB00872 & 3 & Conivaptan & -8.6 \\
\hline DB01126 & 1 & Dutasteride & -8.6 \\
\hline DB01126 & 3 & Dutasteride & -8.6 \\
\hline DB04703 & 1 & Hesperidin & -8.6 \\
\hline DB09280 & 1 & Lumacaftor & -8.6 \\
\hline DB13751 & 3 & Glycyrrhizic acid & -8.6 \\
\hline DB14703 & 0 & Dexamethasone metasulfobenzoate & -8.6 \\
\hline DB14703 & 2 & Dexamethasone metasulfobenzoate & -8.6 \\
\hline DB14703 & 3 & Dexamethasone metasulfobenzoate & -8.6 \\
\hline DB14703 & 4 & Dexamethasone metasulfobenzoate & -8.6 \\
\hline DB00696 & 2 & Ergotamine & -8.5 \\
\hline DB01126 & 0 & Dutasteride & -8.5 \\
\hline DB03147 & 3 & Flavin adenine dinucleotide (FAD) & -8.5 \\
\hline DB06210 & 1 & Eltrombopag & -8.5 \\
\hline DB00637 & 3 & Astemizole & -8.4 \\
\hline DB00696 & 1 & Ergotamine & -8.4 \\
\hline DB00696 & 3 & Ergotamine & -8.4 \\
\hline DB00878 & 0 & Chlorhexidine & -8.4 \\
\hline DB01251 & 1 & Gliquidone & -4 \\
\hline
\end{tabular}


Table 2: Residues involved in hydrogen bond and hydrophobic interaction

\begin{tabular}{|c|c|c|c|}
\hline $\begin{array}{l}\text { Drug Bank } \\
\text { ID }\end{array}$ & Name & H-bond contacts & Hydrophobic Contacts \\
\hline DB14703 & $\begin{array}{l}\text { Dexamethasone } \\
\text { metasulfobenzoate }\end{array}$ & D90, N104, N252, L253 & $\begin{array}{l}\text { V91, E92, G93, Q145, F146, W186, } \\
\text { F190, Q254, N266, H268, D273, }\end{array}$ \\
\hline DB00872 & Conivaptan & & $\begin{array}{l}\text { M58, D90, V91, E92, G93, N104, F146, } \\
\text { W186, A187, F190, N252, L253, Q254, } \\
\text { N266, H268, D273, }\end{array}$ \\
\hline DB01126 & Dutasteride & G93, N104, Q254 & E92, F190, N252, N266, H268, D273, \\
\hline DB04703 & Hesperidin & $\begin{array}{l}\text { D90, V91, E92, Q145, } \\
\text { E191, N252, D273(3) }\end{array}$ & $\begin{array}{l}\text { H148, F146, W186, A187, F190, L253, } \\
\text { N266, H268, A267 }\end{array}$ \\
\hline DB09280 & Lumacaftor & $\begin{array}{l}\text { D90, G93(2), N104, } \\
\text { N252, Q254, N266, }\end{array}$ & $\begin{array}{l}\text { V91, P141, F146, W186, A187, F190, } \\
\text { H268 }\end{array}$ \\
\hline DB13751* & Glycyrrhizic acid & $\begin{array}{l}\text { D90, E92, G93(2), } \\
\text { N104, E191 }\end{array}$ & $\begin{array}{l}\text { V91, Q145, F146, F190, N252, L253, } \\
\text { Q254, H268, N266 }\end{array}$ \\
\hline DB00696 & Ergotamine & & $\begin{array}{l}\text { D90, V91, E92, G93, N104, P141, } \\
\text { Q145, F146, W186, F190, E191, N252, } \\
\text { L253, Q254, N266, D273, }\end{array}$ \\
\hline DB03147 & $\begin{array}{l}\text { Flavin adenine } \\
\text { dinucleotide }\end{array}$ & $\begin{array}{l}\text { V91, N104, E191, N252, } \\
\text { L253, Q254, }\end{array}$ & $\begin{array}{l}\text { D90, G93, H95, P141, F146, A187, } \\
\text { F190, D273, }\end{array}$ \\
\hline DB06210 & Eltrombopag & D90, G93(2), N104 & $\begin{array}{l}\text { H95, P141, F146, W186, A187, F190, } \\
\text { E191, N252, Q254, N266, H268, D273, }\end{array}$ \\
\hline DB00637 & Astemizole & D90, N266 & $\begin{array}{l}\text { V91, E92, G93, N104, P141, F146, } \\
\text { W186, A187, F190, N252, Q254, L253, } \\
\text { H268, D273 }\end{array}$ \\
\hline DB00878 & Chlorhexidine & N266 & W186, Q145, N252, L253, H268, D273 \\
\hline DB01251 & Gliquidone & N252, N266 & $\begin{array}{l}\text { D90, E92, H95, N104, P141, Q145, } \\
\text { F146, W186, A187, F190, H268, D273, }\end{array}$ \\
\hline
\end{tabular}

* LigPlus did not work, manually done

Blue - Catalytic residues

\# Number in bracket indicates number of hydrogen bonds formed with the particular amino acid 


\section{Figures:}

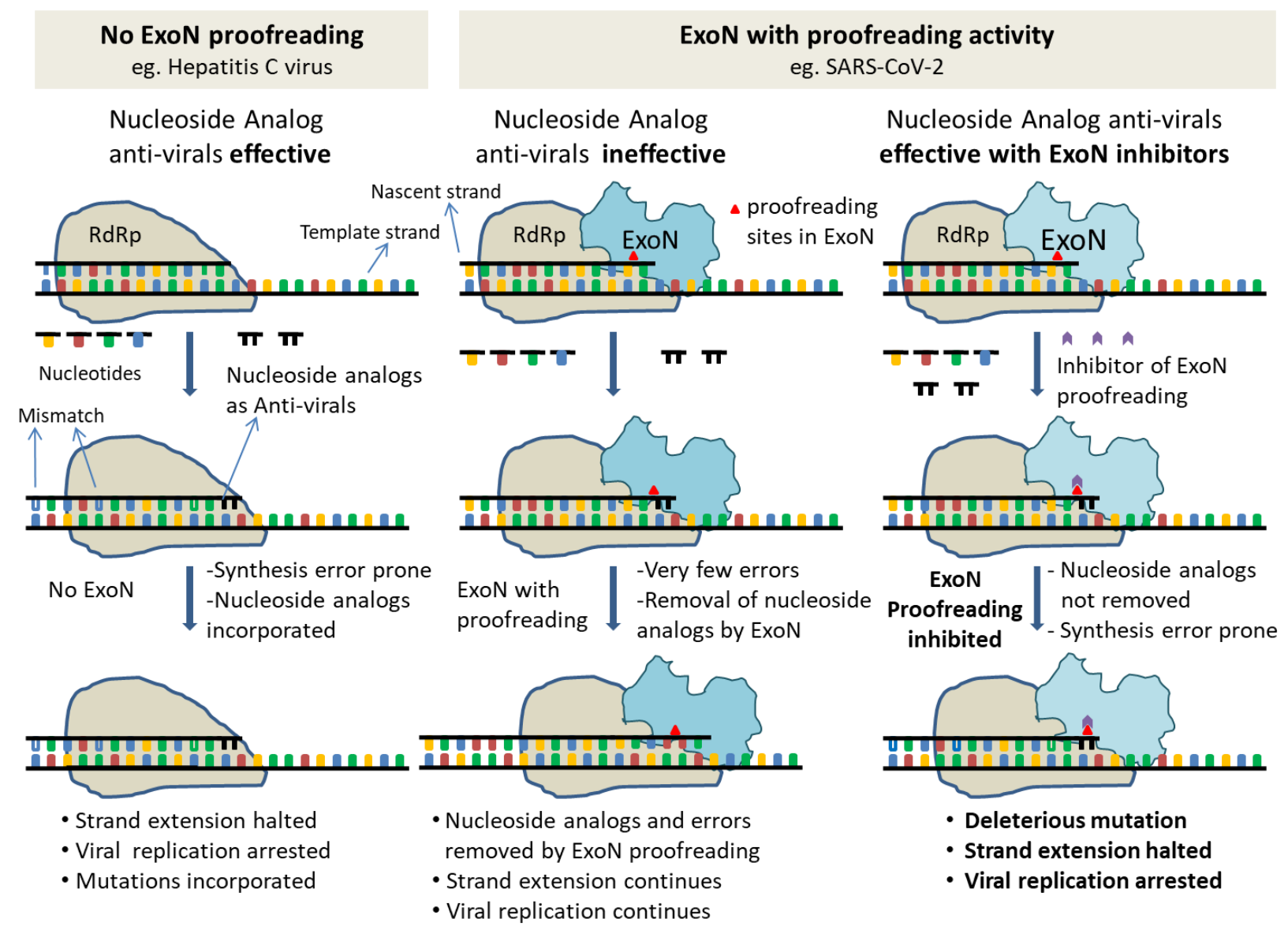

Figure 1: Schematic describing ExoN proofreading activity and mode of action of inhibitors (Left Panel) Replication in viruses like Hepatitis C Virus (HCV), with no proofreading mechanism. Replication by low fidelity $\mathrm{RdRp}$ in absence of ExoN is error prone. Incorporated nucleoside analogs (NA) is not excised resulting in either premature replication termination or incorporation of mutation. (Mid panel) Replication in viruses like SARS-CoV with proofreading Exonuclease. Like HCV, in SARS-CoV-2 too replication is by low fidelity RdRp but errors and NA are excised by proofreading ExoN, decreasing the efficacy of antivirals. (Right panel) Same as mid panel but in presence of ExoN inhibitor. In presence of ExoN inhibitor NA might not be excised, resulting in premature replication termination. 


\section{A}

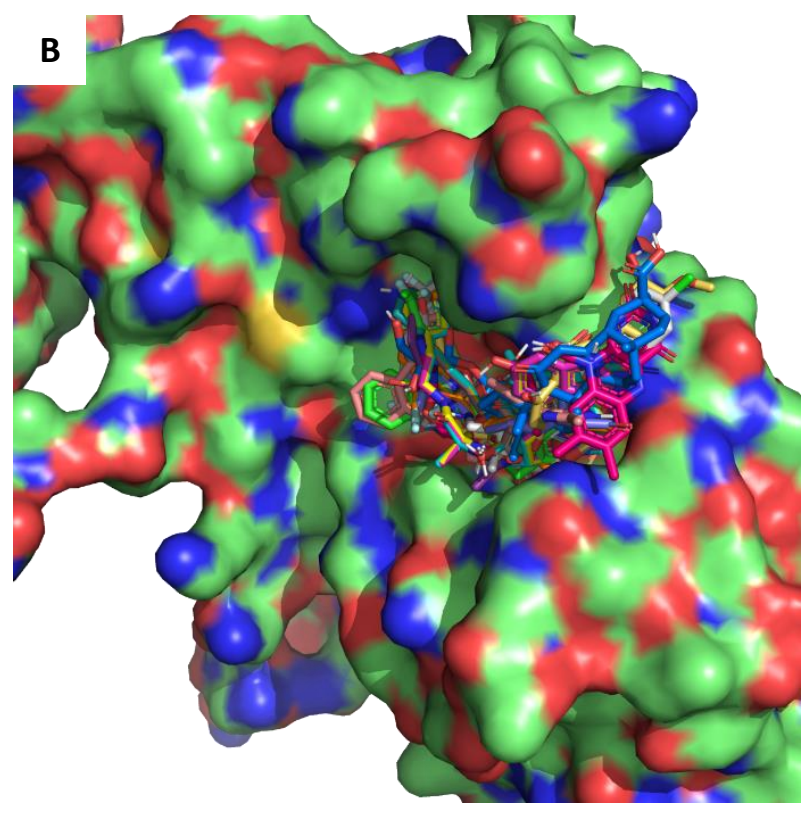

Figure 2: 20 lowest binding energy conformations from the molecular screen

(A) SARS-CoV-2 nsp14 is depicted as surface representation and the 20 lowest binding energy poses are depicted as sticks. The ExoN domain is coloured in green and MTase domain is coloured in blue. (B) Zoomed in version depicting bound conformers of drug molecules. 
A

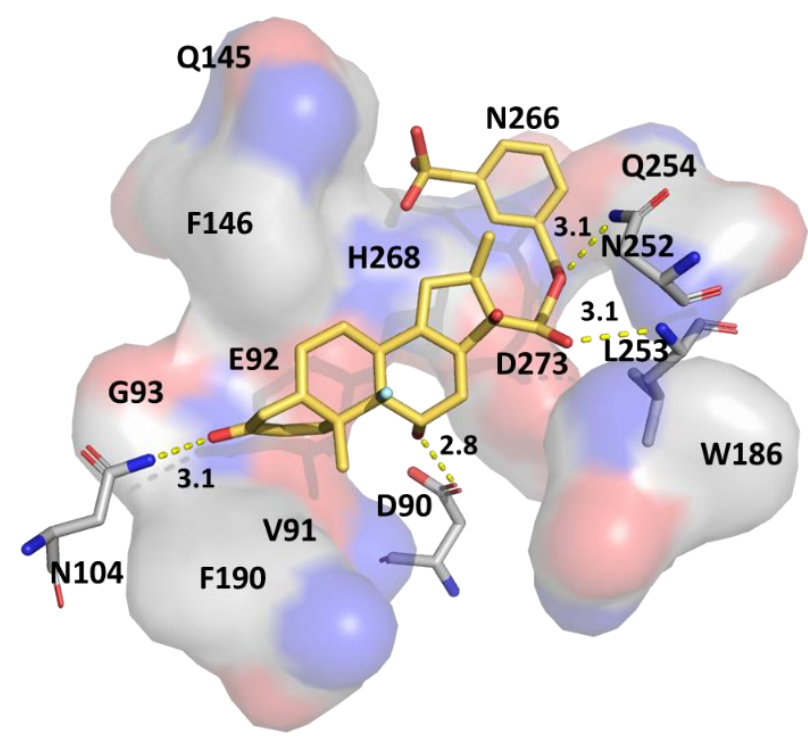

B

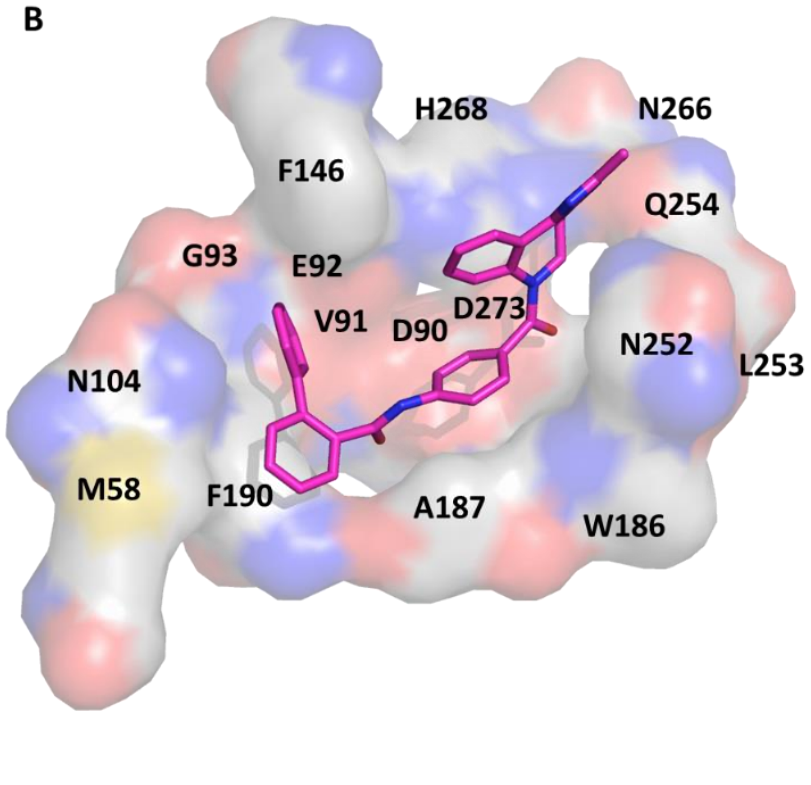

Figure 3: Putative binding pockets of docked compounds

Interaction of ExoN residues (grey colored) with docked conformer of Dexamethasone metasulfobenzoate (yellow sticks) (A) and Conivaptan (pink sticks) (B). H-bonded residues are depicted as sticks. Yellow dotted lines represent hydrogen bond and the distance is in Angstroms. Residues in hydrophobic contact are depicted as surface representation. 


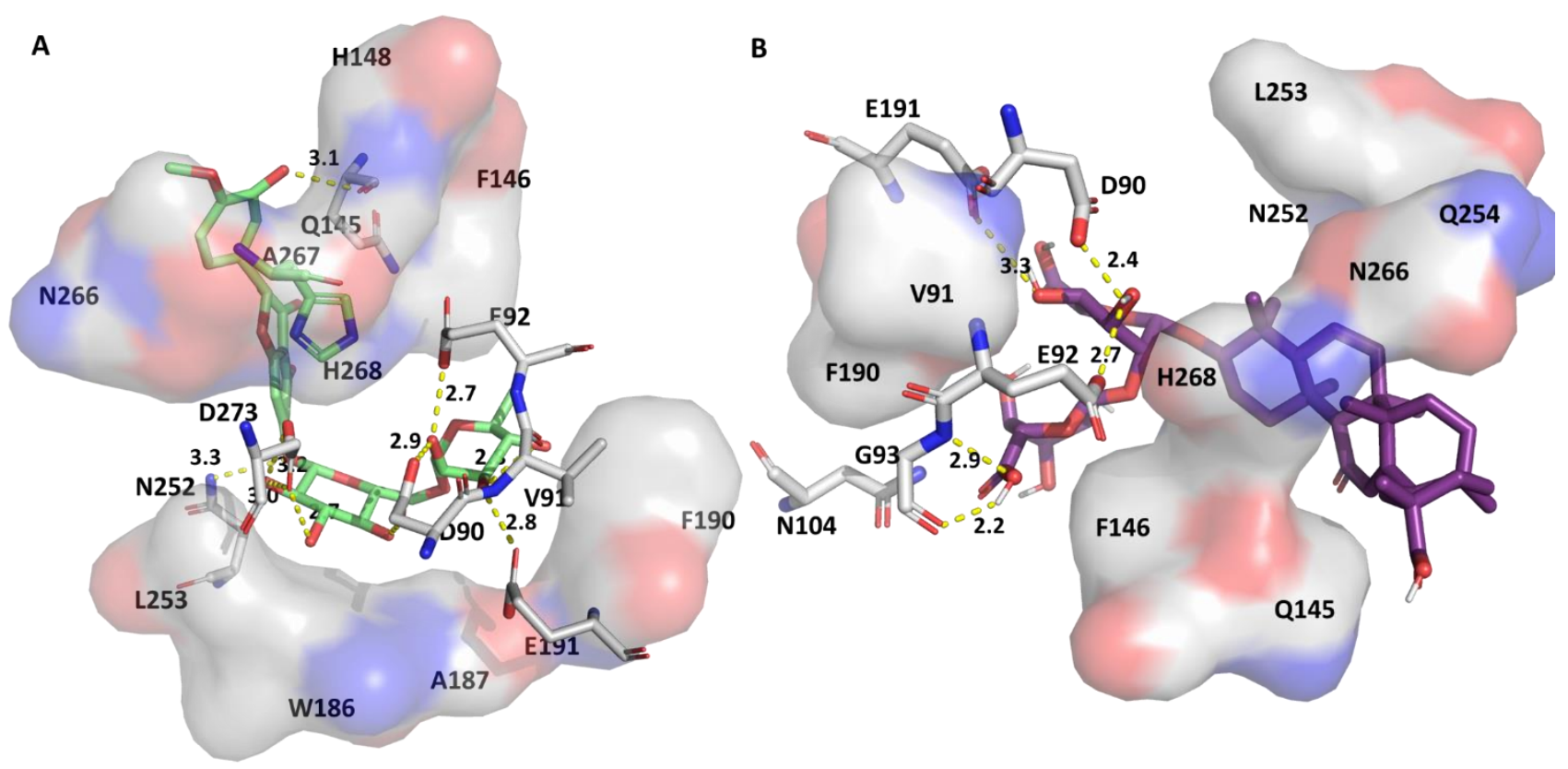

Figure 4: Putative binding pockets of docked compounds:

Interaction of ExoN residues (grey colored) with docked conformer of Hesperidin (green sticks) (A) and Glycyrrhizic acid (purple sticks) (B). H-bonded residues are depicted as sticks. Yellow dotted lines represent hydrogen bond and the distance is in Angstroms. Residues in hydrophobic contact are depicted as surface representation. 


\section{Supplementary Figure:}

\begin{tabular}{|c|c|c|c|c|c|}
\hline \multirow{2}{*}{\multicolumn{2}{|c|}{$\begin{array}{l}\text { Expect } \\
0.0\end{array}$}} & Identities & \multirow{2}{*}{\multicolumn{3}{|c|}{$\begin{array}{l}\text { Positives } \\
520 / 527(98 \%)\end{array}$}} \\
\hline & & $501 / 527(95 \%)$ & & & \\
\hline Query & 1 & \multicolumn{3}{|c|}{$\begin{array}{l}\text { AENVTGLFKDCSKIITGLHPTQAPTHLSVDIKFKTEGLCVDIPGI PKDMTYRRLISMMGF } \\
\text { AENVTGLFKDCSK+ITGLHPTQAPTHLSVD KFKTEGLCVDIPGIPKDMTYRRLISMMGF }\end{array}$} & 60 \\
\hline Sbjet & 1 & \multicolumn{3}{|c|}{ AENVTGLFKDCSKVITGLHPTQAAPTHLSVDTKFKTEGLCVDIPGI PKDMTYRRLISMMGF } & 60 \\
\hline Query & 61 & \multicolumn{3}{|c|}{$\begin{array}{l}\text { KMNYQVNGYPNMFITREEAIRHVRAWIGEDVEGCHATRDAVGTNLPLQLGFSTGVNLVAV } \\
\text { KMNYQVNGYPNMFITREEAIRHVRAWIGFDVEGCHATR+AVGTNLPLQLGFSTGVNLVAV }\end{array}$} & 120 \\
\hline Sbjet & 61 & \multicolumn{3}{|c|}{ KMNYQVNGYPNMFITREEAIRHVRAWIGFDVEGCHATREAVGTNLPLQLGFSTGVNLVAV } & 120 \\
\hline Query & 121 & \multicolumn{3}{|c|}{$\begin{array}{l}\text { PTGYVDTENNTEFTRVNAKPPPGDQFKHLIPLMYKGLPWNVVRIKIVQMLSDTLKGLSDR } \\
\text { PTGYVDT NNT+F+RV+AKPPPGDQFKHLIPLMYKGLPWNVRIKIVQMLSDTLK LSDR }\end{array}$} & 180 \\
\hline Sbjet & 121 & \multicolumn{3}{|c|}{ PTGYVDTPNNTDFSRVSAKP PPGDQFKHLI PLMYKGLPWNVVRIKIVQMLSDTLKNLSDR } & 180 \\
\hline Query & 181 & \multicolumn{3}{|c|}{$\begin{array}{l}\text { VVFVLWAHGFELTSMKYFVKIGPERTCCLCDKRATCFSTSSDTYACWNHSVGFDYVYNPF } \\
\text { VVFVLWAHGFELTSMKYFVKIGPERTCCLCD+RATCFST+SDTYACW+HS+GFDYVYNPF }\end{array}$} & 240 \\
\hline Sbjet & 181 & \multirow{2}{*}{\multicolumn{3}{|c|}{$\begin{array}{l}\text { VVFVLWAHGFELTSMKYFVKIGPERTCCLCDRRATCESTASDTYACWHHSIGFDYVYNPF } \\
\downarrow \\
\text { MIDVQQWGFTGNLQSNHDQHCQVHGNAHVASCDAIMTRCLAVHECFVKRVDWSVEYPIIG } \\
\text { MIDVQQWGFTGNLQSNHD +CQVHGNAHVASCDAIMTRCLAVHECFVKRVDW++EYPIIG }\end{array}$}} & 240 \\
\hline Query & 241 & & & & 300 \\
\hline Sbjet & 241 & \multicolumn{3}{|c|}{ MIDVQQWGFTGNLQSNHDLYCQVHGNAHVASCDAIMTRCLAVHECFVKRVDWTIEYPI IG } & 300 \\
\hline Query & 301 & \multicolumn{3}{|c|}{$\begin{array}{l}\text { DELRVNSACRKVQHMVVKSALLADKFPVLHDIGNPKAIKCVPQAEVEWKFYDAQPCSDKA } \\
\text { DEL++N+ACRKVQHMVVK+ALLADKFPVLHDIGNPKAIKCVPQA+VEWKFYDAQPCSDKA }\end{array}$} & 360 \\
\hline Sbjet & 301 & \multicolumn{3}{|c|}{ DELKINAACRKVQHMVVKAALLADKFPVLHDIGNPKAIKCVPQADVEWKFYDAQPCSDKA } & 360 \\
\hline Query & 361 & \multicolumn{3}{|c|}{$\begin{array}{l}\text { YKIEELFYSYATHHDKFTDGVCLFWNCNVDRYPANAIVCRFDTRVLSNLNLPGCDGGSLY } \\
\text { YKIEELFYSYATH DKFTDGVCLFWNCNVDRYPAN+IVCRFDTRVLSNLNLPGCDGGSLY }\end{array}$} & 420 \\
\hline Sbjet & 361 & \multicolumn{3}{|c|}{ YKIEELFYSYATHSDKFTDGVCLFWNCNVDRYPANSIVCRFDTRVLSNLNLPGCDGGSLY } & 420 \\
\hline uery & 421 & \multicolumn{3}{|c|}{$\begin{array}{l}\text { VNKHAFHTPAFDKSAFTNLKQLPFFYYSDSPCESHGKQVVSDIDYVPLKSATCITRCNLG } \\
\text { VNKHAFHTPAFDKSAF NLKQLPFFYYSDSPCESHGKQVVSDIDYVPLKSATCITRCNLG }\end{array}$} & 480 \\
\hline Sbjet & 421 & \multicolumn{3}{|c|}{ VNKHAFHTPAFDKSAFVNLKQLPFFYYSDSPCESHGKQVVSDIDYVPLKSATCITRCNLG } & 480 \\
\hline Query & 481 & $\begin{array}{l}\text { GAVCRHHANEYR } \\
\text { GAVCRHHANEYR }\end{array}$ & $\begin{array}{l}\text { AGFSLWIYKQFDTYNLWNTFTRLQ } \\
\text { AGFSLW+YKQFDTYNLWNTFTRLQ }\end{array}$ & 527 & \\
\hline - & 481 & GAVCRHHANEYR & AGFSLWVYKQFDTYNLWNTFTRLQ & 527 & \\
\hline
\end{tabular}

Supplementary Figure S1: Pairwise alignment of nsp14 from SARS-CoV and SARSCoV-2 Alignment of SARS-CoV nsp14 (Query) and SARS-CoV-2 nsp14 (Subject) to depict conservation of catalytic residues (highlighted in green). Arrow depicts end of ExoN domain and start of methyl transferase domain. 